Aktuelle Beiträge zum öffentlichen Recht

herausgegeben von:

Rolf Gröschner

Martin Morlok

Helmuth Schulze-Fielitz

Band 1 


\section{Selbsteintrittsrecht der Bauaufsichtsbehörde}

Art. 81 BayB0 und seine Vereinbarkeit mit der gemeindlichen Planungshoheit

Christoph von Damm

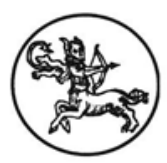

Centaurus Verlag \& Media UG 1998 
Der Autor, Christoph von Damm, studierte Jura an den Universitäten von Würzburg und Bristol (England), 1997 Promotion in Würzburg. Derzeit ist er als Rechtsreferendar in München tätig.

Die Deutsche Bibliothek - CIP-Einheitsaufnahme

Damm, Christoph von:

Selbsteintrittsrecht der Bauaufsichtsbehörde : Art. 81 BayBO und seine Vereinbarkeit mit der gemeindlichen Planungshoheit / Christoph von Damm. - Pfaffenweiler : Centaurus-Verl.-Ges., 1998

(Aktuelle Beiträge zum öffentlichen Recht ; Bd. 1)

Zugl.: Würzburg, Univ., Diss., 1997

ISBN 978-3-8255-0201-0 ISBN 978-3-86226-463-6 (eBook)

DOI 10.1007/978-3-86226-463-6

\section{ISSN $0941-4363$}

Alle Rechte, insbesondere das Recht der Vervielfältigung und Verbreitung sowie der Übersetzung, vorbehalten. Kein Teil des Werkes darf in irgendeiner Form (durch Fotokopie, Mikrofilm oder ein anderes Verfahren) ohne schriftliche Genehmigung des Verlages reproduziert oder unter Verwendung elektronischer Systeme verarbeitet, vervielfältigt oder verbreitet werden.

(C) CENTAURUS-Verlagsgesellschaft mit beschränkter Haftung, Pfaffenweiler 1998

Satz: Vorlage des Autors 
Meinen Eltern! 


\section{Inhaltsverzeichnis}

Seite

Abkürzungsverzeichnis ............................................................................ XIV

1. Teil Einführung ................................................................................................................... 1

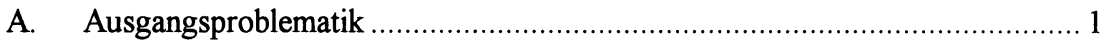

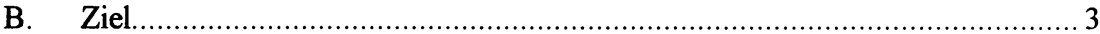

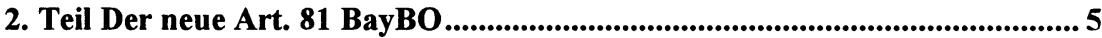

A. Das Beschleunigungsverfahren als Mittel zur Erreichung des Zweck ,schlankerer“ Staat ................................................... 5

B. Art. 81 BayBO als Teil einer umfangreichen Modifizie-

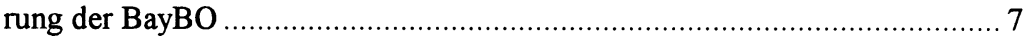

I. Die historische Entwicklung des Bauordnungsrecht bis

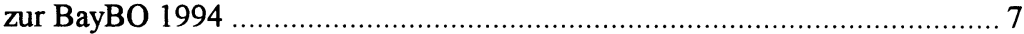

II. Die neuen Regelungen durch die BayBO 1994 ........................................ 9

1. Genehmigungsfreistellung nach Art. 70 BayBO ...................................... 9

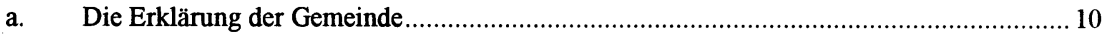

b. Bewertung und Auswirkungen des Erklärungsrechts auf die gemeindliche Planungshoheit im Bauaufsichts- und Baugenehmi-

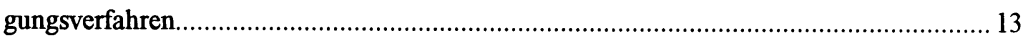

2. Weitere wesentliche Änderungen durch die BayBO

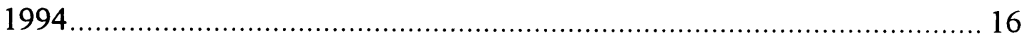

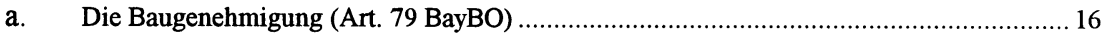

b. Vereinfachtes Genehmigungsverfahren (Art. 80 BayBO) ...............................................16

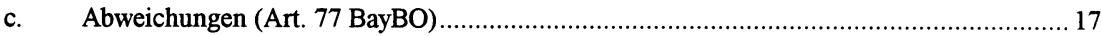

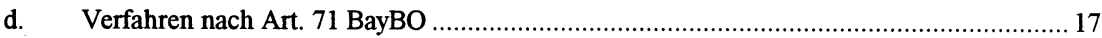

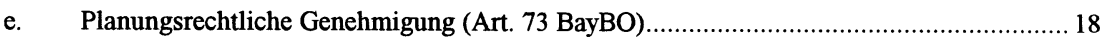

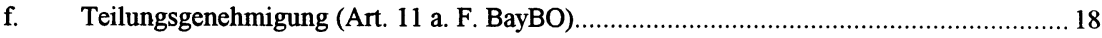

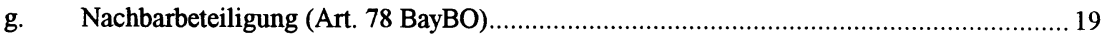

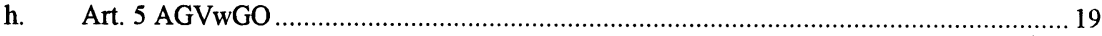

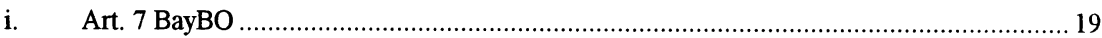

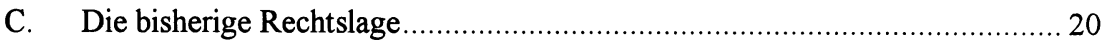

I. Rechtswidrige Versagung des Einvernehmens im Bauggenehmigungsverfahren durch die Gemeinde............................................ 20

II. Bindung an die Versagung des gemeindlichen Einver-
nehmens 


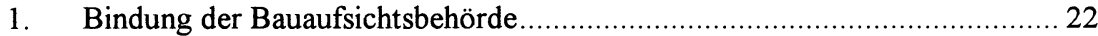

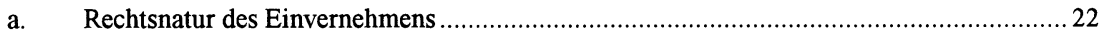

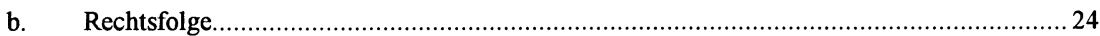

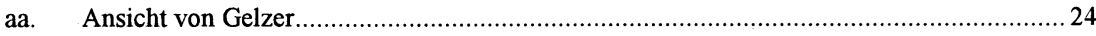

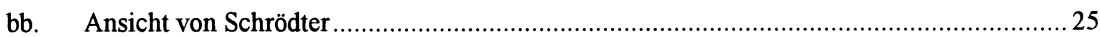

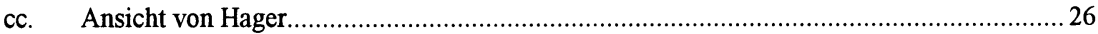

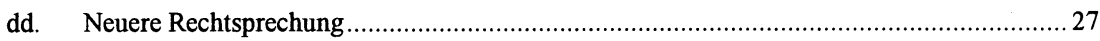

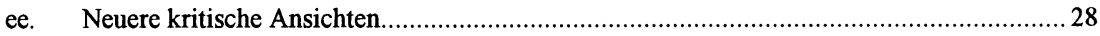

2. Bindung der Widerspruchsbehörde .................................................. 30

3. Bindung der Verwaltungsgerichte .............................................. 31

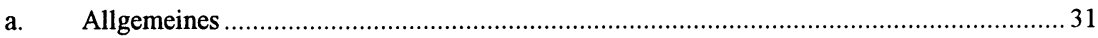

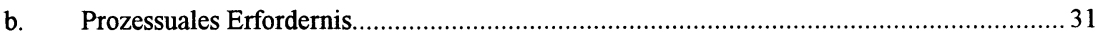

4. Bindungswirkung auch bei einer rechtswidrigen Erteilung des gemeindlichen Einvernehmens............................................ 33

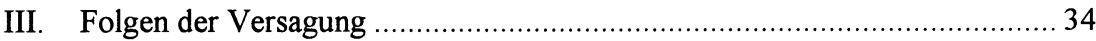

1. Aufsichtliche Ersetzung durch die Rechtsaufsichtsbe-

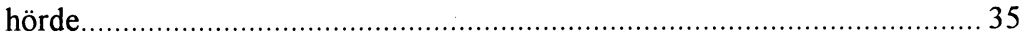

a. Abgrenzung der Kommunalaufsicht zur Bauaufsicht .................................................. 35

b. Aufgaben und Befugnisse der Staatsaufsicht bezogen auf die Er-

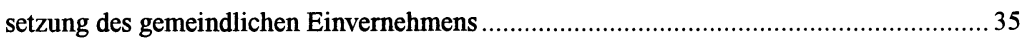

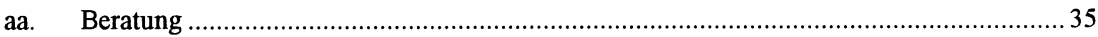

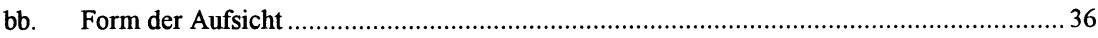

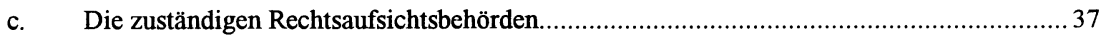

d. Verfahren bei der rechtsaufsichtlichen Ersetzung des gemeindli-

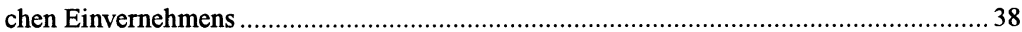

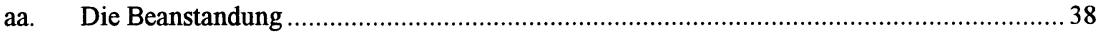

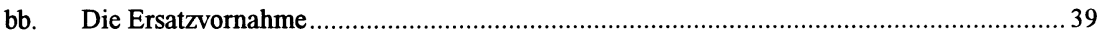

2. Rechtsaufsichtsliche Ersetzung durch die Wider-

IV. Haftung der Gemeinde bei rechtswidrig versagtem Ein-

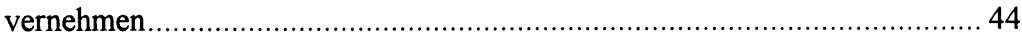

1. Amtshaftung der Gemeinde aus $\S 839$ BGB i. V. $\mathrm{m}$.

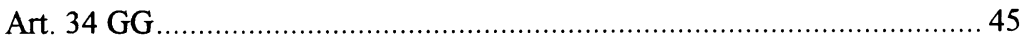

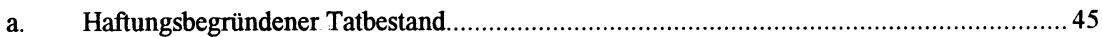

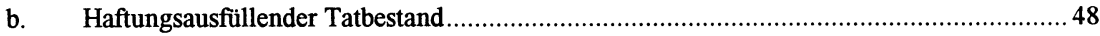

2. Anspruch aus enteignungsgleichem Eingriff ................................. 50

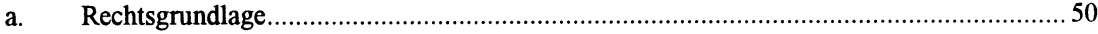

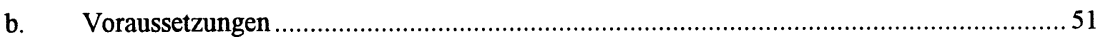

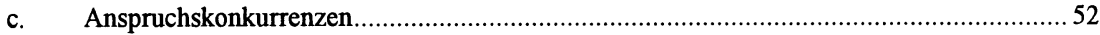

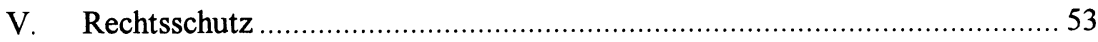

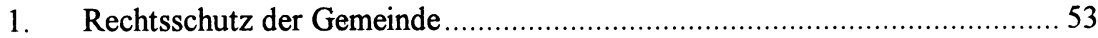


a. Erteilung der Baugenehmigung trotz versagtem Einvernehmen....................................53

b. Rechtsaufsichtliches Ersetzen des gemeindlichen Einvernehmens ...............................55

2. Rechtsschutz des Bauherrn ......................................................... 57

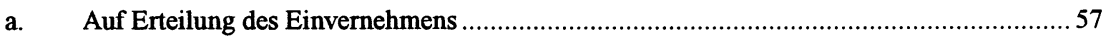

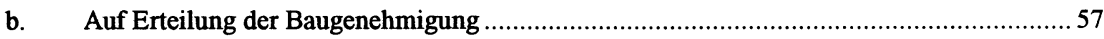

3. Rechtsschutz des Nachbarn ..................................................... 58

a. Gegen die Ersetzung des gemeindlichen Einvernehmens...........................................58

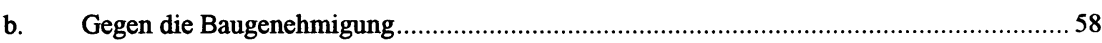

D. Ersetzung des gemeindlichen Einvernehmens nach

Art. 81 Abs. 1-4 BayBO .................................................................. 59

I. Gemeinde i. S. d. Art. 81 Abs. 1 BayBO - das System der

Bauaufsichtsbehörden.................................................................... 59

II. Das Einvernehmens als gemeindlicher Mitwirkungsakt............................6 60

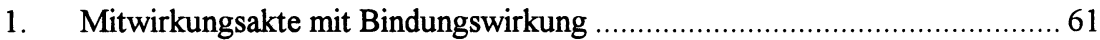

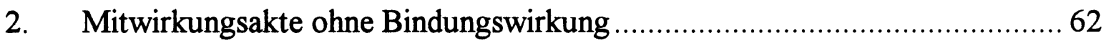

III. Die gesetzlichen Anwendungsfälle des Art. 81 Abs. 1

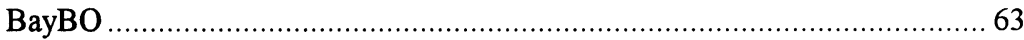

1. Bauplanungsrechtliche Einvernehmen nach $\S 36$ Abs. 1

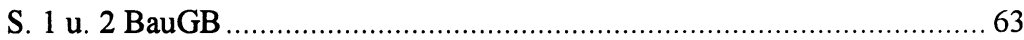

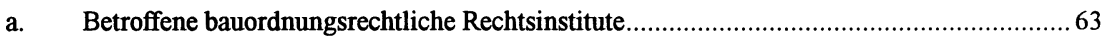

b. Unmittelbar betroffene bauplanungsrechtliche Vorhabendes $\S 36$

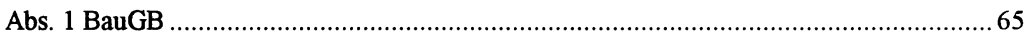

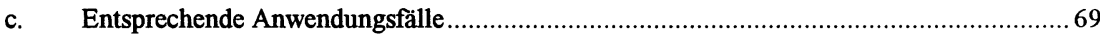

d. Andere öffentlich rechtliche Verfahren i. S. d. $\S 36$ Abs. 1 S. 2

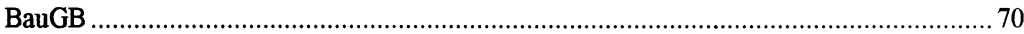

e. Wegfall des Zustimmungserfordernisses nach $\S 36$ Abs. $1 \mathrm{~S} .3$ a.

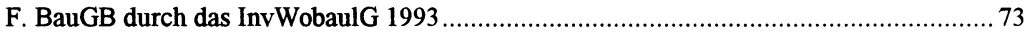

f. Verzicht der Gemeinden auf das Einvernehmenserfordernisses?..................................... 74

2. Ausnahme von einer Veränderungssperre gem. $§ 14$

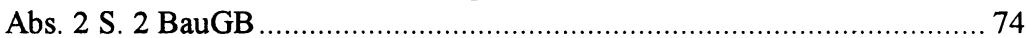

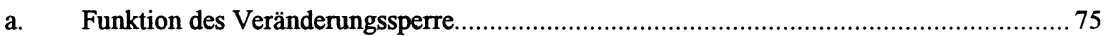

b. Gemeindliches Einvernehmen als Voraussetzung für eine Aus-

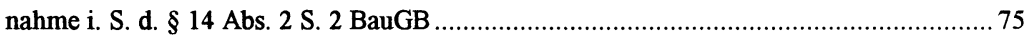

3. Die Teilungsgenehmigung gem. $\S 19$ Abs. 3 BauGB .............................. 76

a. Städtebauliche Erforderlichkeit der Teilungsgenehmigung ........................................... 77

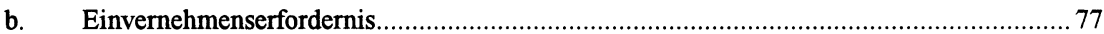

4. Genehmigungen für Wohneigentum in Fremdenver-

kehrsgemeinden gem. $§ 22$ Abs. 6 S. 1 BauGB ................................... 78

5. Bauordnungsrechtliches Einvernehmen bei Abweichun-

gen von örtlichen Bauvorschriften gem. Art. 77 Abs. 2

S. 1 BayBO 
a. Systematik der Abweichungsregel

b. Gemeindliches Einvernehmen bei Abweichungen von örtlichen

Bauvorschriften 80

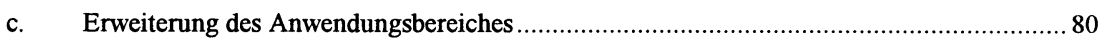

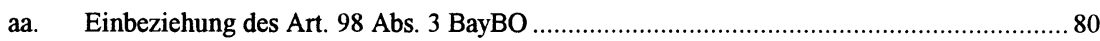

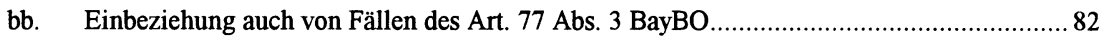

6. Anwendungsfälle über den Wortlaut des Art. 81 Abs. 1 BayBO hinaus ........................................................................ 82

7. Einvernehmen nach mehreren Vorschriften ....................................... 83

8. Ausnahmen vom Einvernehmenserfordernis ..................................... 83

a. Fiktion des Einvernehmens durch Fristablauf............................................................. 83

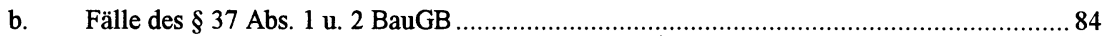

c. Bestandskräftige Teilungsgenehmigung bzw. bestandskräftiger

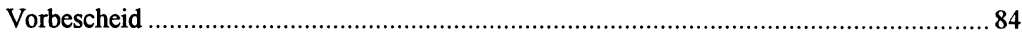

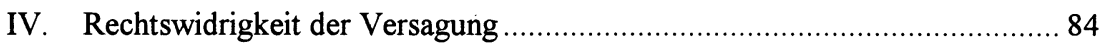

1. Gebundene Entscheidung der Gemeinde ............................................ 85

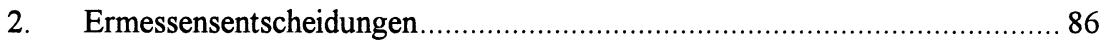

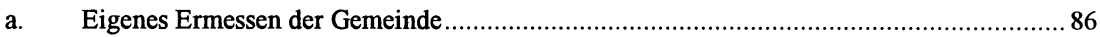

b. Städtebauliche Vertretbarkeit“ in den $\S \S 31$ Abs. 2 Nr. 2 und 34

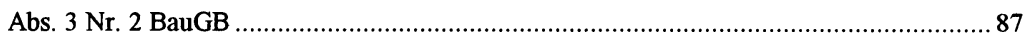

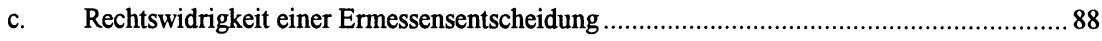

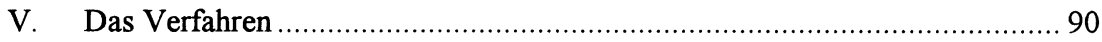

1. Zuständige Ersetzungsbehörde ....................................................... 90

2. Die Terminologie „Ist zu ersetzen“ ............................................... 92

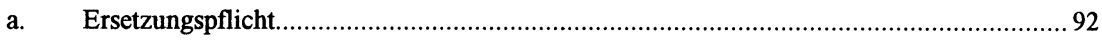

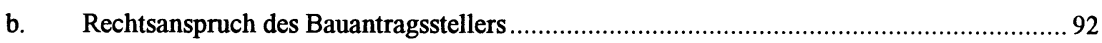

c. Amtspflicht der Bauaufsichtsbehörde aus Art. 81 BayBO ................................................ 93

3. Anhörung der Gemeinde als Ersatz der Beanstandung gem. Art. 112 BayGO ........................................................... 95

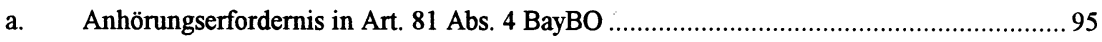

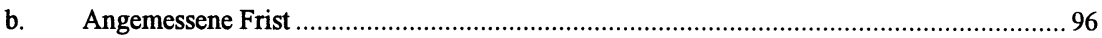

c. Rechtsnatur der erneuten Entscheidung der Gemeinde ................................................. 97

d. Zuständigkeit innerhalb der Gemeinde bei der erneuten Entscheidung.

aa. Abgrenzung der Zuständigkeit des 1. Bürgermeisters und des

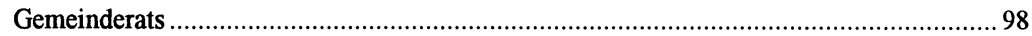

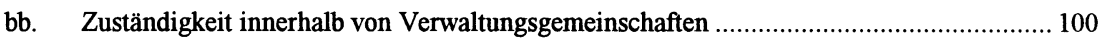

cc. Befangenheit bei gemeinderatlichen Entscheidungen über das

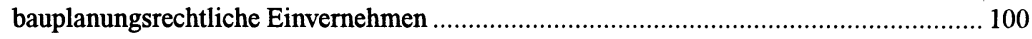

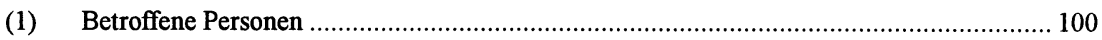

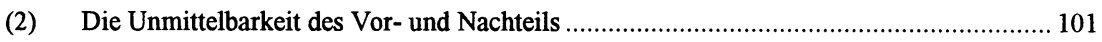


5. Der Doppelverwaltungsakt des Art. 81 Abs. 3 BayBO.

a. Die rechtsdogmatische Einordnung der Genehmigung gem.

Art. 81 Abs. 3 BayBO 102

b. Weitere Voraussetzungen für die Erteilung der Genehmigung......................................... 104

E. Ersetzung des gemeindlichen Einvernehmens nach Art. 93 Abs. 3 i.V.m. Art. 81 BayBO.

F. Ersetzung des gemeindlichen Einvernehmen durch die Widerspruchsbehörde nach Art 81 Abs. 5 BayBO................................ 106

I. Anwendungsbereich ............................................................... 106

II. Kompetenz des Landesgesetzgebers für eine Vorschrift des bundesrechtlich geregelten Widerspruchsverfahrens....................... 109

III. Besonderheiten durch das Widerspruchsverfahren............................... 110

IV. Die Entscheidung durch die Widerspruchsbehörde ............................. 111

F. Rechtsschutz im Fall des Art. 81 BayBO .......................................... 113

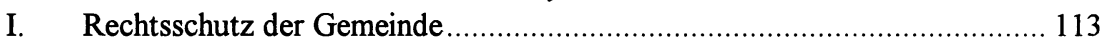

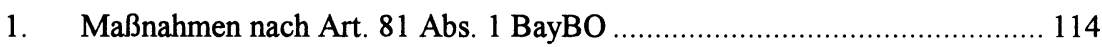

2. Maßnahmen nach Art. 81 Abs. 5 BayBO ............................................. 117

3. Einstweilige Rechtsschutzmöglichkeiten der Gemeinde ........................ 118

a. Aussetzung der aufschiebenden Wirkung - Problem des $\S 10$ Abs. 2 BauGBMaßnahmenG ...................................................................................... 118

b. Rechtsschutz gegen die sofortige Vollziehbarkeit der Genehmigung i. S. d. Art. 81 Abs. 3 BayBO.................................................. 121

II. Rechtsschutz des Bauherrn ...................................................... 122

III. Rechtsschutz des Nachbarn ...................................................... 123

H. Die Überleitungsvorschrift des Art. 100 Abs. 2 BayBO ......................... 125

3. Teil Vereinbarkeit des Art. 81 BayBO mit der gemeindlichen Planungshoheit .......................................................... 126

A. Materielle Verfassungsmäßigkeit des Art. 81 BayBO .......................... 126

I. Eingriff in den Schutzbereich des Selbstverwaltungsrechts aus Art. 28 Abs. 2 GG und A 11 Abs. 2, 83

Abs. $1 \mathrm{BV}$.....

1. Verhältnis der Selbstverwaltungsrechte aus Art. 28

Abs. 2 GG und Art. 11 Abs. 2, 83 Abs. 1 BV

2. Inhalt und Wesen des Selbstverwaltungsrechts

3. Gemeindliche Planungshoheit als verfassungsrechtliche

Verankerung des Selbstverwaltungsrechts

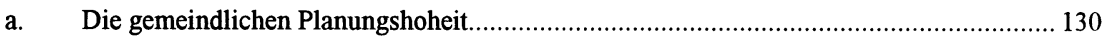

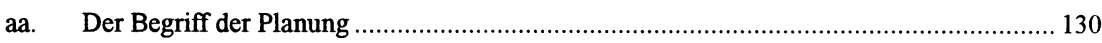

bb. Der Begriff der gemeindlichen Planungshoheit.......................................................... 130 
cc. Konkrete normative Ausgestaltung der gemeindlichen Planungs-

hoheit.

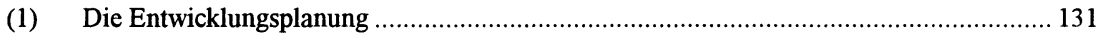

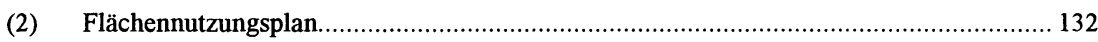

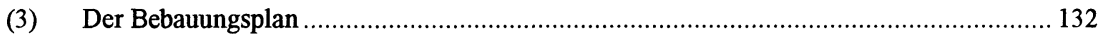

(4). Schutz der noch nicht oder nur unvollständig ausgeübten gemeindlichen Planungshoheit.

b. 1. Stufe des Eingriffs in den Schutzbereich: Gemeindliche Planungshoheit als Angelegenheiten der örtlichen Gemeinschaft ...................................... 135

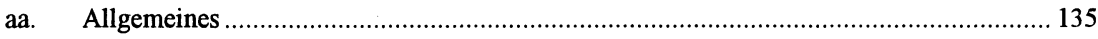

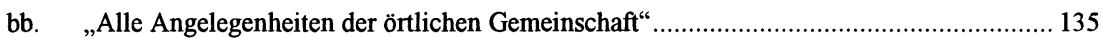

cc. Historische Entwicklung der gemeindlichen Planungshoheit ....................................... 136

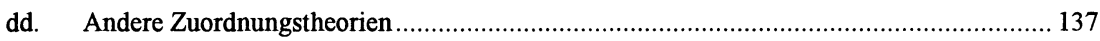

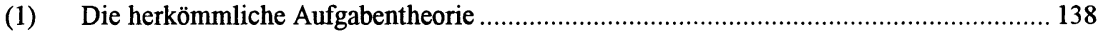

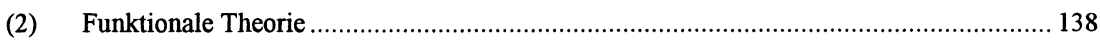

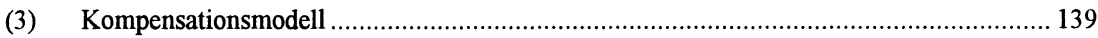

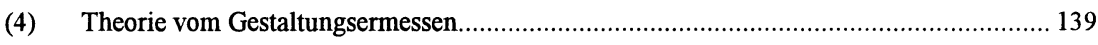

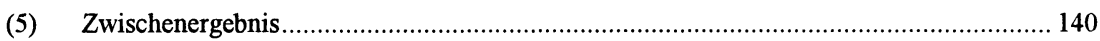

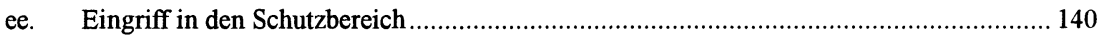

c. 2. Stufe des Eingriffs: Gemeindliche Planungshoheit als Teil des

Kernbereichs gemeindlicher Selbstverwaltung.......................................................... 141

aa. Bestimmung des Kernbereichs des Selbstverwaltungsrechts........................................ 141

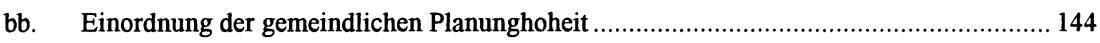

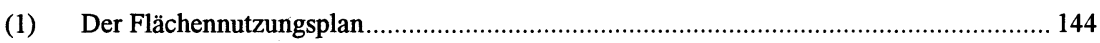

(2) Der Bebauungsplan bzw. der gemeindliche Schutz beim nicht

II. Verfassungsmäßigkeit des Eingriffs ........................................... 147

1. Die rechtlichen Grenzen des Eingriffs ........................................... 147

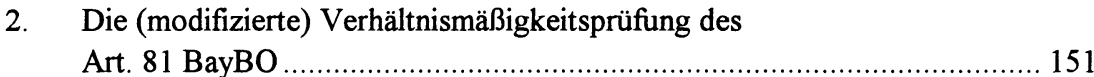

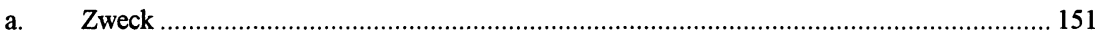

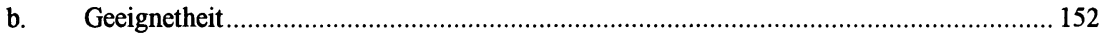

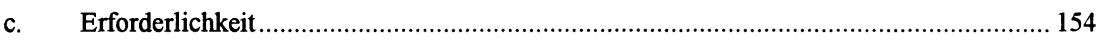

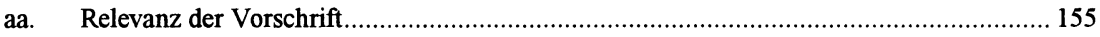

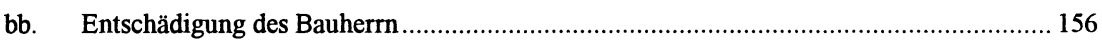

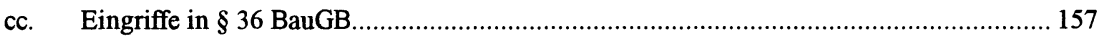

dd. Verfahrensvereinfachungen nach der alten Rechtslage .................................................. 158

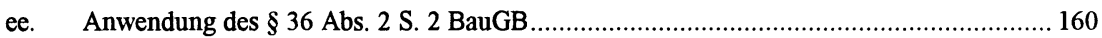

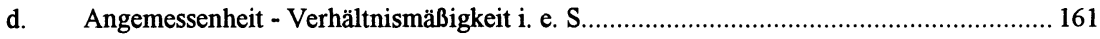

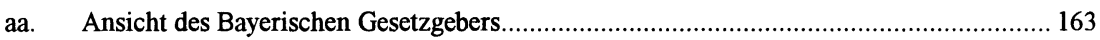

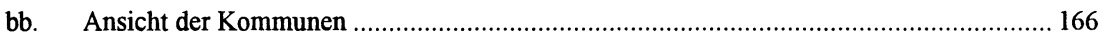




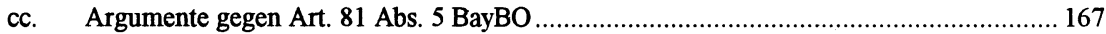

dd. Argumente gegen die Einordnung der Ersetzung in die BayBO ................................... 168

ee. Auswirkungen auf die Praxis und die Gesetzgebung in anderen

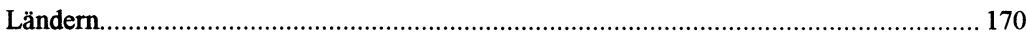

e. Bewertung der Verhältnismäßigkeitsprüfung........................................................... 172

B. Bundeskonformität des Art. 81 BayBO mit § 36 BauGB ........................ 173

I. Verlagerung der Klägerrolle ........................................................ 173

II. Unzulässige Zuständigkeitsverschiebung ..................................... 175

III. Beeinträchtigung der gemeindlichen Befugnisse ............................. 176

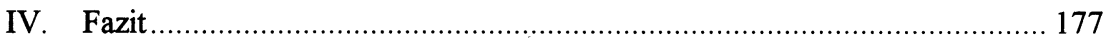

C. Prozessuale Überlegungen ........................................................... 178

I. Kommunale Verfassungsbeschwerde der Gemeinden nach Art. 93 Abs. 1 Nr. 4b GG i. V. m. § 91 BVerfGG vor dem Bundesverfassungsgericht. ............................................ 178

1. Beschwerdeberechtigung und -grund .......................................... 178

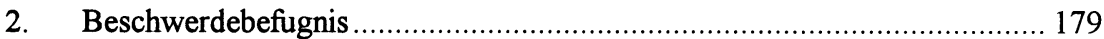

3. Subsidiaritätsklausel ( $₫ 91$ S. 2 BVerfGG) ...................................... 180

4. Beschwerdefrist..................................................................... 182

II. Die bayerische Verfassungsbeschwerde nach Art. 120 BV .................................................................................. 182

III. Die Popularklage der Gemeinden nach Art. 98 S. 4 BV i. V. m. Art. 2 Nr. 7, 55 VerfGHG................................................ 183

1. Allgemeine Voraussetzungen...................................................... 183

2. Popularklagebefugnis................................................................ 184

a. Rechtsschutzfähigkeit des Art. 11 Abs. 2 BV i. S. d. Art. 98 S. 4

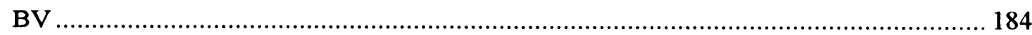

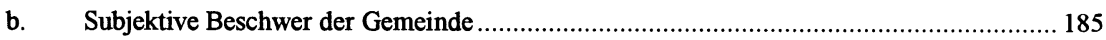

4. Teil Zusammenfassung................................................................... 187

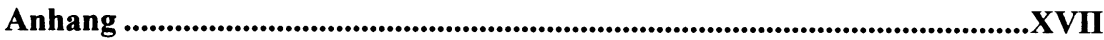

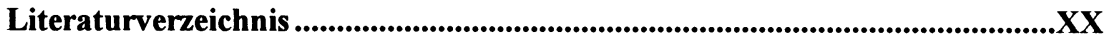




\section{Abkürzungsverzeichnis}

a. A

a. F.

Abs.

Alt

AöR

Art.

BW

BWLBO
AfK

anderer Ansicht

alte Fassung

Absatz

Archiv für Kommunalwissenschaften $(Z)$

Alternative

Archiv des öffentlichen Rechts $(Z)$

Artikel

Baden-Württemberg

Baden-Württembergische Landesbauordnung

BauGB .................................... Baugesetzbuch

BauGBMaßnahmenG

BauNVO

BayBgm

BayBO

BayGO.

BayLtag

BayVBI

$\mathrm{BbgBO}$

BbgGO

Bd.

Beschl.

BGB

BGH

BGHZ

BImSchG

BRD

BRS

BV

BVerfG

BVerfGG

BVerwG.

BVerwGE

bzgl.

bzw.

d. h.
BaugesetzbuchMaßnahmengesetz

Baunutzungsverordnung

Der Bayerische Bürgermeister

Bayerische Bauordnung

Bayerische Gemeindeordnung

Bayerische Landtag

Bayerische Verwaltungsblätter

Brandenburgische Bauordnung

Brandenburgische Gemeindeordnung

Band

Beschluß

Bürgerliches Gesetzbuch

Bundesgerichtshof

Entscheidungen des Bundesgerichtshofs in Zivilsachen

Bundesimmissionsschutzgesetz

Bundesrepublik Deutschland

Baurechtssammlung

Bayerische Verfassung

Bundesverfassungsgericht

Bundesverfassungsgerichtsgesetz

Bundesverwaltungsgericht

Bundesverwaltungsgerichtsentscheidung bezüglich

beziehungsweise

das heißt 


\begin{tabular}{|c|c|}
\hline 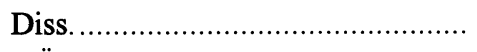 & Dissertation \\
\hline . & Die öffentliche Verwaltung (Z) \\
\hline DVBl . & Deutsches Verwaltungsblatt $(\mathrm{Z})$ \\
\hline EBO ................. & Einheitsbauordnung \\
\hline Einl. .... & Einleitung \\
\hline einschl. . & einschließlich \\
\hline Entsch. . & Entscheidung \\
\hline Erglief. & Ergänzungslieferung \\
\hline etc. & . et cetera \\
\hline f. & folgende \\
\hline 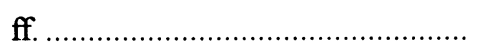 & fortfolgende \\
\hline 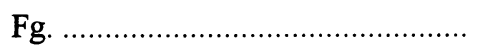 & Festgabe \\
\hline 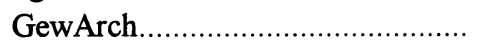 & . Gewerbearchiv (Z) \\
\hline GG & Grundgesetz \\
\hline ggf. & gegebenenfalls \\
\hline grds.... & grundsätzlich \\
\hline GrKrV ... & $\begin{array}{l}\text { Verordnung über Aufgaben der Großen } \\
\text { Kreisstädte }\end{array}$ \\
\hline GVBI & Gesetzes- und Verordnungsblatt \\
\hline HessBauO ....... & Hessische Bauordnung \\
\hline hinsichtl....... & hinsichtlich \\
\hline HS....... & Hauptsatz \\
\hline 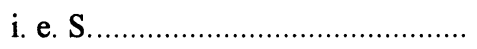 & im engeren Sinne \\
\hline i. d. R. & in der Regel \\
\hline 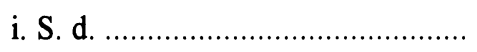 & im Sinne des \\
\hline i. V. m. & in Verbindung mit \\
\hline JA & Juristische Arbeitsblätter (Z) \\
\hline JuS. & Juristische Schulung (Z) \\
\hline 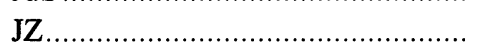 & Juristische Zeitung (Z) \\
\hline $\mathrm{KG} \ldots \ldots \ldots \ldots \ldots$ & Kostengesetz \\
\hline LBO. & Landesbauordnung \\
\hline LKrO & Landkreisordnung für den Freistaat Bayern \\
\hline LKV & Landes- und Kommunalverwaltung $(\mathrm{Z})$ \\
\hline m. E. . & meines Erachtens \\
\hline MBO & Musterbauordnung \\
\hline 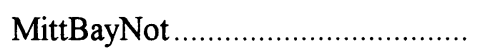 & Mitteilungen der Bayerischen Notare \\
\hline n. F. & neue Fassung \\
\hline NJW & Neue Juristische Wochenschrift (Z) \\
\hline 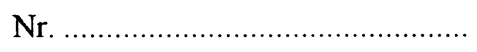 & Nummer \\
\hline NuR ... & Natur und Recht $(\mathrm{Z})$ \\
\hline NVwZ.. & Neue Zeitschrift für Verwaltungsrecht $(Z)$ \\
\hline
\end{tabular}


NVwZ-RR

NWGO

NWVBI
NVwZ-Rechtsprechungsreport (Z)

Nordrhein-Westfälische Gemeindeordnung

Nordrhein-Westfälische Verwaltungsblätter (Z)

OVG

Pr. ALR

Rn.

Rspr.

S.

SächsBO

SchlHLbO

Oberverwaltungsgericht

Algemeines Preußisches Landrecht

Randnummer

Rechtsprechung

Seite

Sächsische Bauordnung

Schleswig-Holsteinische Landesbauordnung

sog. sogenannt

$\mathrm{StGBBr}$ Städte- und Gemeindebund Brandenburg

$\mathrm{StGH}$ Staatsgerichtshof

unveröff. unveröffentlicht

UPR

Umwelt- und Planungsrecht

Urt. Urteil

u. U. unter Umständen

UVPG Gesetz über die Umweltverträglichkeitsprüfung V. von, vom

VblBW Verwaltungsblätter Baden-Württemberg

Verf. Verfassung

VerfGHG Gesetz über den Bayerischen Verfassungsgerichtshof

VerwRspr Verwaltungsrechtsprechung $(Z)$

VfGHG

Gesetz über den rheinland-pfälzischen Verfassungsgerichtshof

VG. Verwaltungsgericht

VGH Verfassungsgerichtshof

vgl.

Vorb vergleiche

VR

Vorbemerkung

Verwaltungsrundschau $(Z)$

VerwArch

Verwaltungsarchiv $(\mathrm{Z})$

VwGO

Verwaltungsgerichtsordnung

VwVfG

Verwaltungsverfahrensgesetz

z. B. zum Beispiel

ZustVBau zit. (Z) Zuständigkeitsverordnung im Bauwesen zitiert

Zeitschrift

Im übrigen gelten die Abkürzungen des „Kirchnerverzeichnisses“ 